\title{
EIN BRIEF VON ABEL AN DEGEN.
}

In der Kopenhagener Universitätsbibliothek habe ich vor einigen Jahren einen Brief von Nils HenRik Aber an den dänischen Mathematiker Professor Cari Furdinand Degen (1766-i 825) gefunden und habe ihn in der Norsk Matematik Tidsskrift 1935 publiziert. Dieser Brief ist in mehrfacher Hinsicht von grossem Interesse, denn er stammt aus einer Periode in Abels Leben, für welche das historische Material sehr dürftig ist, und er enthält wichtige Einzelheiten für Abels Lebens- und Entwicklungsgeschichte. Bis jetzt habe ich gezögert, eine deutsche Utbersetzung mit den nötigen Anmerkungen zu bringen, weil ich als Geometer mich nicht kompetent gefühlt habe, den mathematischen Inhalt gründlich genug zu beleuchten. Nun hat aber Professor EPstein in Frankfurt a. M. in dankenswerter Weise meiner Bitte, dies zu tun, Folge geleistet und hat seine Bemerkungen zum Abdruck zur Verfügung gestellt. Seine Bemerkungen folgen auf den Brief von Abel.

Was die in dem Briefe erwähnten Personen betrifft, so genügt es, folgendes mitzuteilen: A rgustcs KreJdal (I 790-I 829) war ein dänischer Gymnasiallehrer, der mathematische Lehrbücher und philosophische Abhandlungen verfasste. Henrik Gerner von Schmidten (I 799-I83I) wurde i 827 Nachfolger von Degen an der Kopenhagener Universität und Erasmus Georg Fog Thune (I785-i 829 ) im Jahre I8I 5 Nachfolger von Bugge für Astronomie und Mathematik.

Poul Heeganrl.

Christiania den 2ten März I 824.

Herrn Professor Degen.

Sehr wohl fühle ich, Herr Professor, dass ich Ihrer Verzeihung bedarf, weil ich nicht früher an Sie geschrieben habe und Ihnen meinen besten Dank bezeugt habe für die besondere Zuvorkommenheit and Bereitwilligkeit, womit Sie mich während meines Aufenhaltes in Kopenhagen behandelt haben, was ich gewiss niemals vergessen, sondern woran ich mich stets mit der grössten Dankbarkeit erinnern werde. Ich habe viele Male im Sinne gehabt, Herrn Professor zu schreiben,

1--36808. Acta mathematica. 68. Imprimé le 12 mars 1937. 
aber ich habe das von einer zur anderen Zeit verschoben, da ich den Wunsch hatte, gleichzeitig eine kleine Abhandlung mitzuschicken, und ich habe erst in der letzten Zeit eine fertig gebracht, die ich als nur einigermassen Threr Beurteilung würdig ansehen kann; dass sie dies ganz ist, darf ich nicht hoffen. Sofern sich eine Gelegenheit findet, werde ich mir die Freiheit nehmen, sie Ihnen zu schicken. - Seit ich die Ehre hatte, Herrn Professors lehrreichen Umgang zu geniessen, habe ich mich besonders mit der Integralrechnung beschäftigt, und ich darf vielleicht sagen, nicht ohne jedes Glück: Ich habe wiederum den ersten 'Teil der Integralrechnung durchgearbeitet, die ich ausgearbeitet hatte, bevor ich nach Kopenhagen reiste und der ich so glücklich war eine, wie ich glaube, ziemlich systematische Form zu geben. Ich hatte die Hoffnung, dass ich sie hier in Christiania auf Kosten der Universität drucken lassen könnte, aber da ich zur selben Zeit für ein Reisestipendium vorgeschlagen wurde und man ausserdem glaubte, dass meine Abhandlung eine passendere Stelle in den Schriften einer wissenschaftlichen Gesellschaft finden würde, so wurde nichts aus der Sache. Gott weiss, wo ich sie gedruckt bekomme, und ich wünschte das so von Herzen, da ich glaube, dass eigene Arbeiten die beste Empfehlung für mich sein würden bei meinem Auslandsaufenhalt, von dem ich Grund habe zu glauben, dass er in einem Jahr oder so ungefähr seinen Anfang nehmen wird. - Was die Franzosen comparaison des transcendantes nennen, damit habe ich mich sehr viel beschäftigt. Und da diese in enger Verbindung steht mit der vollständigen algebraischen Auf. lösung der Differentialgleichungen, $\varphi(y) d y+\varphi(x) d x=0$, so habe ich eine allgemeine Methode gesucht und gefunden, um die Form zu bestimmen, die die Funktion $\varphi$ haben muss, damit das vollständige Integral dieser Gleichung in einer algebraischen Form ausgedrïckt werden kann. Ich habe vorausgesetzt, dass $\varphi$ eine algebraische Function bezeichnet. Ich habe die allgemeine Methode angewandt auf die durch Eulers und Lagranges Untersuchungen bekannte separierte Differentialgleichung

$$
\frac{d y}{\sqrt{a+a_{1} y+\cdots+a_{m} y^{m}}}+\frac{d x}{\sqrt{a+a_{1} x+\cdots+a_{m} x^{m}}}=0 .
$$

Ich bin zu dem Resultat gekommen, dass man um diese Gleichung algebraisch aufzulösen, die Gleichung

$$
\left(\frac{\partial \nu}{\partial y}\right)=\frac{\mathrm{I}}{2} \varphi\left(a_{1}+2 a_{2} x+\cdots+m a_{m} x^{m-1}\right)+\left(\frac{\partial \varphi}{\partial x}\right)\left(a+a_{1} x+\cdots+a_{m} x^{m}\right)
$$


erfüllen können muss, wo $\nu$ und $\varphi$ zwei rationale und symmetrische Functionen von $x$ und $y$ bezeichnen. Die Auflösung dieser Gleichung ist, wie Herr Professor sieht, mehr weitläufig als schwierig. Hat man diese Gleichung erfüllt, so ist

$$
c=\nu+\varphi \sqrt{a+a_{1} y+\cdots+a_{m} y^{m}} \cdot \sqrt{a+a_{1} x+\cdots+a_{m} x^{m}}
$$

das vollständige Integral der voranstehenden Differentialgleichung; $c$ ist constante arbitraire. - So weit mir bekannt, sind alle transcendenten Functionen, deren Eigenschaften man bis jetzt durch eine endliche Gleichnng zwischen zwei Variablen ausdruicken konnte, in der Form enthalten

$$
\int \frac{p d x}{\sqrt{a+a_{1} x+a_{2} x^{2}+a_{3} x^{3}+a_{4} x^{4}}}
$$

oder können durch eine bequeme Substitution auf diese Form gebracht werden. Ich bin durch ein zufälliges Ereignis dazu gekommen, dass ich eine Eigenschaft von allen transcendenten Funktionen $\int \varphi(z) d z$, wo $\varphi(z)$ eine ganz beliebige algebraische irrationale Funktion von $z$ bedeutet, ausdrücken kann durch eine solche Gleichung, und zwar zwischen so vielen veränderlichen Grössen, wie man will; nämlich wenn man bezeichnet $\int \varphi(z) d z=\psi(z)$, so kann man immer eine Gleichung von der Form

$$
\psi\left(z_{1}\right)+\psi\left(z_{2}\right)+\psi\left(z_{3}\right)+\cdots+\psi\left(z_{n}\right)=\psi\left(\alpha_{1}\right)+\psi\left(\alpha_{2}\right)+\cdots+\psi\left(\alpha_{n}\right)+p
$$

finden, wo $z_{1}, z_{2}$, etc. algebraische Funktionen von einer beliebigen Anzahl veränderlicher Grössen sind ( $n$ ist abhängig von dieser Anzahl und im Allgemeinen sehr viel grösser); $\alpha_{1}, \alpha_{2}, \ldots$ sind constante Grössen und $p$ ist eine algebraische und logarithmische Funktion; sie ist in vielen Fällen gleich o. Dieses Theorem und eine Abhandlung darüber habe ich gedacht an das Institut de France zu schicken, da es, wie mir scheint, Licht verbreiten wird über die transcendenten Funktionen im Allgemeinen. Von allen transcendenten Funktionen habe ich mir jedoch besondere Mühe mit den elliptischen Transcendenten gegeben, da ich stets Herrn Professors Rath an mich in einem Brief an Professor Hansteen vor Augen gehabt habe. Ich habe mich deswegen bestrebt, eine »Théorie des transcendantes elliptiques» auszuarbeiten, in der ich so weit es mir möglich war versucht habe die Methode zu zeigen, der man folgen muss, um diese interessante und überaus nützliche Theorie so vollkommen zu machen, wie es, nach dem augenblicklichen 
Stand der Analysis möglich ist; aber das ist wie gesagt nur ein Versuch und ich bin weit davon entfernt zu glauben, dass diese Theorie vollkommen ist, was sie wohl kaum in Jahrhunderten werden wird. - Indem ich über die elliptischen Transcendenten spreche, kann ich mich nicht enthalten zu äussern, dass Legendre eine allgemeinere Methode besitzen muss, als die er in seinen Untersuchungen vorlegt. Es finden sich wirklich Stellen bei ihm, die das sind, was Herr Kreidal vom Hinmel gefallen nennt und zu denen man, wie es scheint, kaum kommen kann ohne eine allgemeine Methode. Ich glaube, Herr Professor ist mit mir der Meinung, dass kaum etwas schädlicher für den Fortschritt der Wissenschaft ist, als so die Methode zu verbergen. - Am Schluss dieser meiner Theorie habe ich einen Gegenstand behandelt, um den man sich, wie es scheint, bis jetzt nicht gekümmert hat, nämlich um die verschiedenen Werte, die die elliptischen Transcendenten haben, denn ebenso wie z. B. der Logarithmus einer Grösse unendlich viele Werthe hat, so ist es auch naheliegend, dass die elliptischen Transcendenten dieselbe Eigenschaft haben. Ich bin zu dem Resultat gekommen, dass wenn $p$ einen Werth für eine elliptische Transcendente bezeichnet, die übrigen von der Form $\pm n a \pm m b+p$ sind, wo $a$ und $b$ konstante Grössen bedeuten, die im Allgemeinen imaginär sind und deren Verhältnis nicht rational ist. $m$ und $n$ bezeichnen beliebige ganze Zahlen. Jede elliptische Transcendente hat so unendlich mal unendlich viele (sit venia verbo) Werte. - In der Theorie der sogenannten intégrales définies habe ich mich auch geübt, und da nun das Allgemeine ein grosses Interesse für mich hat, so suchte ich, ob ich eine Formel finden könnte, von der Legendres schönes Theorem

$$
\frac{\pi}{2}=F^{1}(c) \cdot E^{1}(b)+F^{1}(b) \cdot E^{1}(c)-F^{1}(c) \cdot F^{1}(b)
$$

ein besonderer Fall ist. Ich fand so folgende Formel:

$$
\begin{aligned}
& \frac{C}{\left(a-a^{2}\right)^{1+\frac{1}{2} n+\frac{1}{2} m}}=\int \frac{d \varphi(\cos \varphi)^{m}(\sin \varphi)^{n}}{\left(1-a \sin ^{2} \varphi\right)^{m+\frac{1}{2} n+\frac{1}{2}}} \int \frac{d \varphi(\cos \varphi)^{m}(\sin \varphi)^{n+2}}{\left(1-(1-a) \sin ^{2} \varphi\right)^{m+\frac{1}{2} n+\frac{3}{2}}} \\
& +\int \frac{d \varphi(\cos \varphi)^{m}(\sin \varphi)^{n}}{\left(\mathrm{I}-(\mathrm{I}-a) \sin ^{2} \varphi\right)^{m+\frac{1}{2} n+\frac{1}{2}}} \int \frac{d \varphi(\cos \varphi)^{m}(\sin \varphi)^{n+2}}{\left(\mathrm{I}-a \sin ^{2} \varphi\right)^{m+\frac{1}{2} n+\frac{3}{2}}} .
\end{aligned}
$$

Die Integrale sind von $\varphi=0$ b1s $\varphi=\frac{\pi}{2}$ genommen. $\quad C$ ist eine constante Grösse unabhängig von $a$ und $m$ und $n$ beliebig. Nimmt man $m=n=0$, so bekommt man 
eine Formel, aus der man leicht Legendres Formel ableiten kann. Diese ist also ein sehr specieller Fall der obenstehenden. - Den beiliegenden Brief an Herrn von Schmidten wage ich Herrn Professor zu ersuchen zu besorgen; ich kenne seine Adresse nicht. Sollte er nicht zurückgekommen sein, so sind Sie vielleicht so gut, ihn zu vernichten. -

Zum Schluss muss ich Herrn Professor bitten, meine Dreistigkeit zu entschuldigen, Sie mit diesem Brief zu beschweren, und sollten Sie in ihm Äusserungen finden, die unpassend scheinen für einen Lehrling und beginnenden Mathematiker, so habe ich aus Unwissenheit gefehlt und rechne in dieser Hinsicht auf Ihre Güte; aber nichts könnte mir unlieber sein als diese zu missbrauchen.

Meine ebrerbietigsten Grüsse an Professor Thune, und Dank für alle bewiesene Güte bitte ich zu bestellen.

\section{Mit Hochachtung Ihr} N. H. Abell.

Bemerkungen zu dem vorstehenden Brief.

von P. Epstein, Frankfurt a/M.

Der Brief von Abel an Degen ist, wie Herr Heegand mit Recht hervorhebt, von grösstem Interesse für ABEcs Lebensgeschichte und gibt wichtige Aufschlüsse über seine mathematische Entwicklung. Er enthält den frühesten Hinweis auf die Periodizitätsmoduln des elliptischen Integrals erster Gattung ${ }^{1}$, ferner die erst 1827 in dem Aufsatz "Sur quelques intégrales définies» (Crelle Bd. 2) veröffentlichte Integralformel, die eine Verallgemeinerung der LEGENDrkschen Relation darstellt ${ }^{2}$, vor allem aber ist darin zum ersten Mal das Abelsche Theorem erwähnt, in dessen Besitz also AвEx bereits in seinem 22. Lebensjahr gewesen ist. Damit wird die Angabe von Herrn Fr. Lange-Ninlsen, »Zur Geschichte des Abelschen Theorems" (Tidsskrift 1927), Aвex habe das Theorem vor seinem vollendeten 23. Jahre entdeckt, bestätigt und präzisiert. Allerdings findet sich in dem vorliegenden Briefe noch nicht der Begriff des Geschlechts als der Mini-

1 Die Bemerknng, dass das Verhältnis der Moduln nicht „rational» ist, könnte überraschen; man erwartet statt rational eher "reell". Dies scheint aber Abel auch in seinen Abhandlungen immer stillschweigend voranszasetzen.

Oeuvres 2. édition, 1, 256 Formel (7). Um die im Brief angegebene Formel zu erhalten, hat man $\beta=\alpha$ zu nehmen und in den Integralen die Substitution $a x=\operatorname{ctg}^{2} \varphi$ bezw. $(1-a) x=\operatorname{ctg}^{2} \varphi$ zu machen and dann $\mathrm{I}-2 \alpha=m, 4 \alpha+2 \gamma-3=n$ za setzen. 
malzahl der Integrale, durch die eine Summe von "Abelschen» Integralen dargestellt werden kann, und man kann mit Bestimmtheit sagen, dass Aber ihn damals noch nicht besessen hat, denn sonst hätte er nicht den Ansatz für ein Additionstheorem von zwei hyperelliptischen Integralen gemacht. Dies deutet darauf hin, dass ABEL, offenbar in Unkenntnis ähnlicher Versuche von EOLER ${ }^{1}$ und $\mathrm{L}_{\mathrm{AGRANGE}}{ }^{2}$, zunächst die Verallgemeinerung des Additionstheorems der elliptischen Integrale in einer Richtung suchte, die nicht zum Ziele führte, und dadurch dann auf sein Theorem hingelenkt wurde.

Die früheste schriftliche Fixierung des Abelschen Theorems lag bis jetzt in dem nachgelassenen Aufsatz Sur la Comparaison des fonctions transcendantes» (Oeuvres 2, 55) vor, und der Beweisansatz ist darin fast wörtlich derselbe, wie in'dèm berühmten, wenige Monate vor'seinem Tode niedergeschriebenen Aufsatz „Démonstration d'une propriété générale... (Crelle Bd. 4, Oeuvres 1, 515). Jenèr nachgelassene Aufsatz ist nach dem Zeugnis von HoLmвов (Oenvres 2, 324) vor der grossen Reise, die ABEL im September 1825 antrat, geschrieben, und in ihm tritt der Begriff des Geschlechts auf (Oeuvres 2, 57). Wir können also die Auffindung dieses wichtigen Begriffes mit Sicherheit auf die Zeit zwischen der $\mathbf{A b}$ fassung des vorliegenden Briefes und dem Antritt der Reise ansetzen. Auch in dem Brief an Crelue vom 9. August 1826 (Oeuvres 2, 267) und in der grossen Pariser Akademieabhandlung (eingereicht am 30. Oktober 1826) findet sich dieser Begriff, ebenso, für den hyperelliptischen Fall, in dem Aufsatz »Remarques sur quelques propriétés générales...» (Cvelle Bd. 3, Oeuvres 1, 454), während er wiederum in der oben erwähnten letzten Arbeit (Crelle Bd. 4) fehlt.

i Novi Comm. Petrop. XII, 1766/67 (Publ. 1768) p. 42-68.

" Misc. Taur. IV, $1766 / 69$ p. $98-125$. 